\title{
On Surface Meshes Induced by Level Set Functions
}

\author{
Maxim A. Olshanskii*, Arnold Reusken ${ }^{\dagger}$, and \\ Xianmin $\mathrm{Xu}^{\dagger \ddagger}$
}

Bericht Nr. 347

Oktober 2012

Key words: surface finite elements, level set function, surface triangulation, maximum angle condition

AMS Subject Classifications: 58J32, 65N15, 65N30

\section{Institut für Geometrie und Praktische Mathematik RWTH Aachen}

\author{
Templergraben 55, D-52056 Aachen (Germany)
}

\footnotetext{
* Department of Mathematics, University of Houston, Houston, Texas 77204-3008 and Department of Mechanics and Mathematics, Moscow State University, Moscow 119899, Russia (molshan@math.uh.edu).

Institut für Geometrie und Praktische Mathematik, RWTH Aachen University, D-52056 Aachen, Germany (reusken@igpm.rwth-aachen.de, xu@igpm.rwth-aachen.de). 


\title{
ON SURFACE MESHES INDUCED BY LEVEL SET FUNCTIONS
}

\author{
MAXIM A. OLSHANSKII*, ARNOLD REUSKEN ${ }^{\dagger}$, AND XIANMIN XU ${ }^{\dagger \ddagger}$
}

\begin{abstract}
The zero level set of a piecewise-affine function with respect to a consistent tetrahedral subdivision of a domain in $\mathbb{R}^{3}$ is a piecewise-planar hyper-surface. We prove that if a family of consistent tetrahedral subdivions satisfies the minimum angle condition, then after a simple postprocessing this zero level set becomes a consistent surface triangulation which satisfies the maximum angle condition. We treat an application of this result to the numerical solution of PDEs posed on surfaces. We show that the nodal basis of a $P_{1}$ finite element space with respect to this surface triangulation is $L^{2}$-stable, provided a natural scaling is used. Furthermore, the issue of stability of the nodal basis with respect to the $H^{1}$-norm is addressed.
\end{abstract}

Key words. surface finite elements, level set function, surface triangulation, maximum angle condition

1. Introduction. Surface triangulations occur in, for example, visualization, shape optimization, surface restoration and in applications where differential equations posed on surfaces are treated numerically. Hence, properties of surface triangulations such as shape regularity and angle conditions are of interest. For example, angle conditions are closely related to approximation properties and stability of corresponding finite element $[1,2]$.

In this note, we are interested in the properties of a surface triangulation if one considers the zero level of a piecewise-affine function with respect to a consistent tetrahedral subdivision of a domain in $\mathbb{R}^{3}$. The zero level of a piecewise-affine function is a piecewise-planar hyper-surface consisting of triangles and quadrilaterals. Each quadrilateral can be divided into two triangles in such a way that the resulting surface triangulation satisfies the following property proved in this paper: if the volume tetrahedral subdivision satisfies a minimum angle condition, then the corresponding surface triangulation satisfies a maximum angle condition. We show that the maximum angle occuring in the surface triangulation can be bounded by a constant $\phi_{\max }<\pi$ that depends only on a stability constant for the family of tetrahedral subdivisions.

The paper also discusses a few implications of this property for the numerical solution of surface partial differential equations. Numerical methods for surface PDEs are studied in e.g., [5, 4, 8, 3, 6, 11]. We derive optimal approximation properties of $P_{1}$ finite element functions with respect to the surface triangulation and a uniform bound for the condition number of the scaled mass matrix. We also show that the condition number of the (scaled) stiffness matrix can be very large and is sensitive to the distribution of the vertices of tetrahedra close to the surface. Some numerical examples illustrate the analysis of the paper.

2. Surface meshes induced by regular bulk triangulations. Consider a smooth surface $\Gamma$ in three dimensional space. For simplicity, we assume that $\Gamma$ is connected and has no boundary. Let $\Omega \subset \mathbb{R}^{3}$ be a bulk domain which contains $\Gamma$. Let $\left\{\mathcal{T}_{h}\right\}_{h>0}$ be a family of tetrahedral triangulations of the domain $\Omega$. These

\footnotetext{
${ }^{*}$ Department of Mathematics, University of Houston, Houston, Texas 77204-3008 and Dept. Mechanics and Mathematics, Moscow State University, Moscow 119899 (molshan@math.uh.edu).

${ }^{\dagger}$ Institut für Geometrie und Praktische Mathematik, RWTH-Aachen University, D-52056 Aachen, Germany (reusken@igpm.rwth-aachen.de,xu@igpm.rwth-aachen.de).

${ }^{\ddagger}$ LSEC, Institute of Computational Mathematics and Scientific/Engineering Computing, NCMIS, AMSS, Chinese Academy of Sciences, Beijing 100190, China (xmxu@lsec.cc.ac.cn).
} 
triangulations are assumed to be regular, consistent and stable. To simplify the presentation we assume that this family of triangulations is quasi-uniform. The latter assumption, however, is not essential for our analysis.

We assume that for each $\mathcal{T}_{h}$ a polygonal approximation of $\Gamma$, denoted by $\Gamma_{h}$, is given with the following properties. $\Gamma_{h}$ is a $C^{0,1}$ surface without boundary and $\Gamma_{h}$ can be partitioned in planar triangular segments. We assume that $\Gamma_{h}$ is consistent with the outer triangulation $\mathcal{T}_{h}$ in the following sense. For any tetrahedron $S_{T} \in \mathcal{T}_{h}$ such that $\operatorname{meas}_{2}\left(S_{T} \cap \Gamma_{h}\right)>0$ define $T=S_{T} \cap \Gamma_{h}$. We assume that every $T \in \Gamma_{h}$ is a planar segment and thus it is either a triangle or a quadrilateral. Each quadrilateral segment can be divided into two triangles, so we may assume that every $T$ is a triangle.

Let $\mathcal{F}_{h}$ be the set of all triangular segments $T$, then $\Gamma_{h}$ can be decomposed as

$$
\Gamma_{h}=\bigcup_{T \in \mathcal{F}_{h}} T .
$$

The most prominent example of such a surface triangulation is obtained in the context of level set techniques. Assume that $\Gamma$ is represented as the zero level of a level set function $\phi$ and that $\phi_{h}$ is a continuous linear finite element approximation on the outer tetrahedral triangulation $\mathcal{T}_{h}$. Then if we define $\Gamma_{h}$ to be the zero level of $\phi_{h}$ then $\Gamma_{h}$ consists of piecewise planar segments and is consistent with $\mathcal{T}_{h}$. As an example, consider a sphere $\Gamma$, represented as the zero level of its signed distance function. For $\phi_{h}$ we take the piecewise linear nodal interpolation of this distance function on a uniform tetrahedral triangulation $\mathcal{T}_{h}$ of a domain that contains $\Gamma$. The zero level of this interpolant defines $\Gamma_{h}$ and is illustrated in Fig. 2.1.
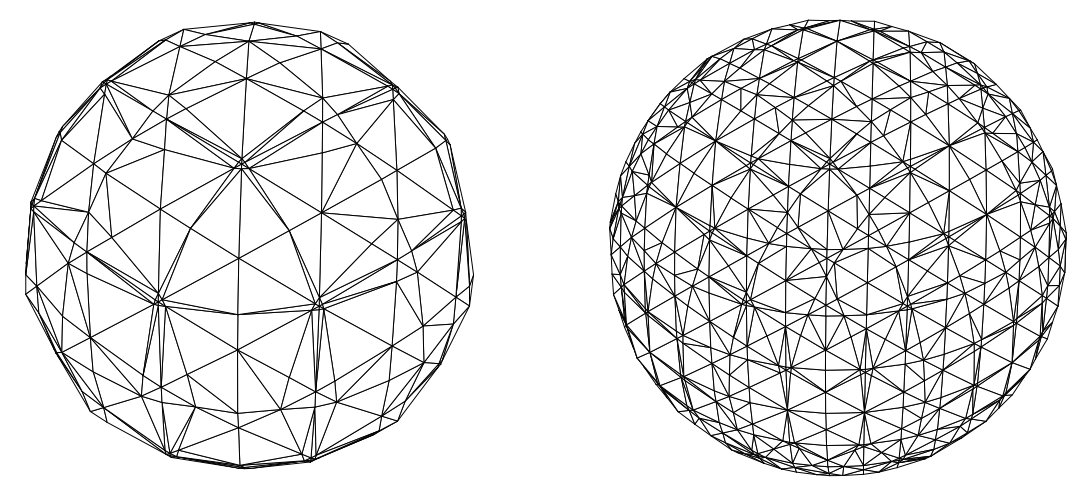

FIG. 2.1. Approximate interface $\Gamma_{h}$ for an example of a sphere, resulting from a coarse tetrahedral triangulation (left) and after one refinement (right).

In the setting of level set methods, such surface triangulations induced by a finite element level set function on a regular outer tetrahedral triangulation are very natural and easy to construct. A surface triangulation $\Gamma_{h}$ that is consistent with the outer triangulation may be the result of another method than the level set method. In the remainder we only need that $\Gamma_{h}$ is consistent to the outer triangulation and not that it is generated by a level set technique.

Note that the triangulation $\mathcal{F}_{h}$ is not necessarily regular, i.e. elements from $T$ may have very small inner angles and the size of neighboring triangles can vary strongly, cf. Fig. 2.1. In the next section we prove that, provided each quadrilateral is divided into two triangles properly, the induced surface triangulation is such that the maximal angle condition [1] is satisfied. 
3. The maximal angle condition. The surface triangulation $\Gamma_{h}=\cup_{T \in \mathcal{F}_{h}} T$ is assumed to be consistent with the outer tetrahedral triangulation $\mathcal{T}_{h}$, as defined in section 2. The family of outer tetrahedral triangulations $\left\{\mathcal{T}_{h}\right\}_{h>0}$ is assumed to be regular, i.e., it contains no hanging nodes and the following stability property holds:

$$
\sup _{h>0} \sup _{S \in \mathcal{T}_{h}} \rho(S) / r(S) \leq \alpha<\infty,
$$

where $\rho(S)$ and $r(S)$ are the diameters of the smallest ball that contains $S$ and the largest ball contained in $S$, respectively. Although the surface mesh $\Gamma_{h}$ induced by $\mathcal{T}_{h}$ can be highly shape irregular, the following lemma shows that a maximum angle property holds.

Lemma 3.1. Assume an outer triangulation $\mathcal{T}_{h}$ from the regular family $\left\{\mathcal{T}_{h}\right\}_{h>0}$ and let $\Gamma_{h}$ be consistent with $\mathcal{T}_{h}$. For any $S \in \mathcal{T}_{h}$ there exists $\phi_{\min }>0$, depending only on $\alpha$ from (3.1), such that:

a) if $T=S \cap \Gamma_{h}$ is a triangle element, then

$$
0<\phi_{i, T} \leq \pi-\phi_{\min } \quad i=1,2,3,
$$

holds, where $\phi_{i, T}$ are the inner angles of the element $T$.

b) if $T=S \cap \Gamma_{h}$ is a quadrilateral element, then

$$
\phi_{i, T} \geq \phi_{\min }, \quad i=1,2,3,4,
$$

holds, where $\phi_{i, T}$ are the inner angles of the element $T$.

Proof. Take $S \in \mathcal{T}_{h}$. Let $\theta_{\min }>0$ be such that all inner angles of all sides of $S$ and all angles between edges of $S$ and their opposite side are in the interval $\left[\theta_{\min }, \pi-\theta_{\min }\right]$. From the stability property it follows that

$$
\frac{\pi}{2}>\theta_{\min } \geq c(\alpha)>0
$$

holds with a constant $c(\alpha)$ that depends only on $\alpha$ from (3.1).

We first treat the case where $T=S \cap \Gamma_{h}$ is a triangle $T=B C D$, as illustrated in Fig. 3.1. Consider the angle $\phi:=\angle B C D$. Then either $\phi \leq \pi-\theta_{\min }$ and (3.2) is

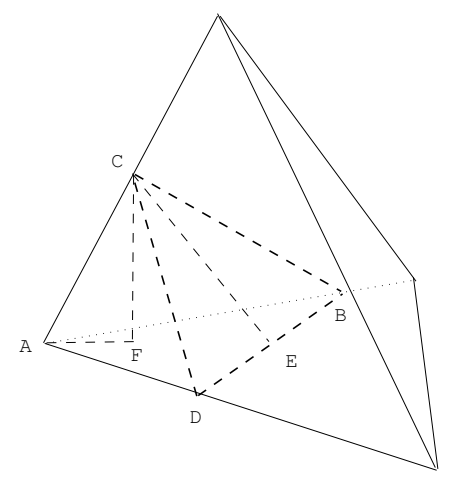

FIG. 3.1.

proved with $\phi_{\min }=\theta_{\min }$ or $\phi \in\left(\pi-\theta_{\min }, \pi\right)$. Hence, we treat the latter case. Note that

$$
\frac{|C F|}{|A C|}=\sin (\angle C A F) \geq \sin \theta_{\min }
$$


and $\angle B D C<\pi-\phi<\theta_{\min }<\frac{\pi}{2}$. Take $E$ on the line through $D B$ such that $C E \perp D B$, and $F$ in the plane through $A B D$ such that $C F$ is perpendicular to this plane. Hence, $|C F| \leq|C E|$ holds. Using the sine rule we get

$$
\begin{aligned}
\sin (\angle A D C) & =\frac{|A C|}{|C D|} \sin (\angle C A D) \leq \frac{|A C|}{|C D|} \leq \frac{1}{\sin \theta_{\min }} \frac{|C F|}{|C D|} \leq \frac{1}{\sin \theta_{\min }} \frac{|C E|}{|C D|} \\
& =\frac{1}{\sin \theta_{\min }} \sin (\angle B D C) \leq \frac{\sin (\pi-\phi)}{\sin \theta_{\text {min }}}=\frac{\sin (\phi)}{\sin \theta_{\text {min }}}<1 .
\end{aligned}
$$

Hence, $\angle A D C \leq \arcsin \left(\frac{\sin \phi}{\sin \theta_{\min }}\right) \leq 2 \frac{\sin \phi}{\sin \theta_{\min }}$ holds. This yields

$$
\angle A D B<\angle A D C+\angle C D B \leq 2 \frac{\sin \phi}{\sin \theta_{\min }}+\pi-\phi .
$$

With the same arguments we obtain

$$
\angle A B D \leq 2 \frac{\sin \phi}{\sin \theta_{\min }}+\pi-\phi .
$$

Since $\angle D A B \leq \pi-\theta_{\min }$ and $\angle D A B=\pi-(\angle A D B+\angle A B D)$ we get

$$
\theta_{\min } \leq 4 \frac{\sin \phi}{\sin \theta_{\min }}+2 \pi-2 \phi
$$

For $\phi \in\left(\pi-\theta_{\min }, \pi\right)$ the inequality (3.4) holds iff $\phi \leq \phi_{0}$, where $\phi_{0}$ is the unique solution in $\left(\frac{1}{2} \pi, \pi\right)$ of $2 \sin \phi_{0}+\left(\pi-\phi_{0}\right) \sin \theta_{\min }=\frac{1}{2} \theta_{\min } \sin \theta_{\min }$. This proves the result in a).

We now consider the case where $T=S \cap \Gamma_{h}$ is a quadrilateral $T=A B C D$, as illustrated in Fig. 3.2. Consider the angle $\phi:=\angle D A B$. Then either $\phi \in\left(0, \theta_{\min }\right)$ or

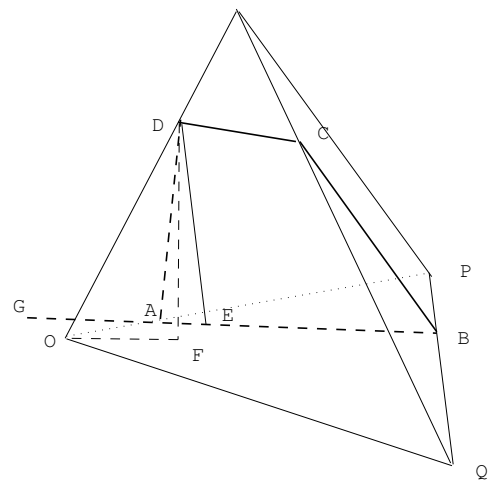

FiG. 3.2 .

$\phi \in\left[\theta_{\min }, \pi\right)$. We only should treat the former case. Take $E$ on the line through $A B$ such that $D E \perp A B$, and $F$ in the plane through $O P Q$ such that $D F$ is perpendicular to this plane. Hence, $|D F| \leq|D E|$ holds and

$$
\sin \phi=\frac{|D E|}{|A D|}
$$


Furthermore, using $\frac{|D F|}{|O D|}=\sin (\angle D O F) \geq \sin \theta_{\min }$ we get

$$
\begin{aligned}
\sin (\angle O A D) & =\frac{|O D|}{|A D|} \sin (\angle A O D) \leq \frac{|O D|}{|A D|} \leq \frac{1}{\sin \theta_{\min }} \frac{|D F|}{|A D|} \\
& \leq \frac{1}{\sin \theta_{\min }} \frac{|D E|}{|A D|}=\frac{\sin \phi}{\sin \theta_{\text {min }}}<1 .
\end{aligned}
$$

This implies

$$
\angle O A D \leq \arcsin \left(\frac{\sin \phi}{\sin \theta_{\min }}\right) \leq 2 \frac{\sin \phi}{\sin \theta_{\min }} .
$$

Hence, since $\angle D A B=\phi \leq 2 \sin \phi$, we obtain

$$
\angle O A B<\angle O A D+\angle D A B \leq\left(1+\frac{1}{\sin \theta_{\min }}\right) 2 \sin \phi .
$$

Using $\angle O A B=\pi-\angle P A B$ and $\angle P A B<\pi-\angle O P Q<\pi-\theta_{\text {min }}$ results in

$$
\theta_{\min }<\left(1+\frac{1}{\sin \theta_{\min }}\right) 2 \sin \phi .
$$

For $\phi \in\left(0, \theta_{\text {min }}\right)$ the inequality (3.5) holds iff $\phi \geq \phi_{0}$, where $\phi_{0}$ is the unique solution in $\left(0, \frac{1}{2} \pi\right)$ of $\theta_{\min }=\left(1+\frac{1}{\sin \theta_{\min }}\right) 2 \sin \phi_{0}$. Thus the result in b) holds.

$\square$

The lemma readily yields the following result.

ThEOREM 3.2. Consider a regular family of tetrahedral triangulations $\left\{\mathcal{T}_{h}\right\}_{h>0}$ and a surface triangulation $\Gamma_{h}=\cup_{T \in \mathcal{F}_{h}} T$ that is consistent to $\mathcal{T}_{h}$. Assume that any quadrilateral element $T=S \cap \Gamma_{h}, S \in \mathcal{T}_{h}$, is divided in two triangles by connecting its vertex with largest inner angle with its opposite vertex. The resulting surface triangulation satisfies the following maximal angle condition. There exists $\phi_{\min }>0$ depending only on $\alpha$ from (3.1) such that:

$$
0<\sup _{T \in \mathcal{F}_{h}} \phi_{i, T} \leq \pi-\phi_{\min } \quad i=1,2,3,
$$

where $\phi_{i, T}$ are the inner angles of the element $T$.

Proof. If $T=S \cap \Gamma_{h}$ is a triangle, then (3.6) directly follows from (3.2). Let $T=S \cap \Gamma_{h}$ be a quadrilateral, with its four inner angles denoted by $\theta_{4} \geq \theta_{3} \geq \theta_{2} \geq$ $\theta_{1}>0$. From the result in (3.3) we have $\theta_{i} \geq \phi_{\min }$ for all $i$. The vertex with angle $\theta_{4}$ is connected with the opposite vertex. Let $T_{1}$ be one of the resulting triangles. One of the angles of $T_{1}$ is $\theta_{j}$ with $j \in\{1,2,3\}$. From $\theta_{j} \geq \phi_{\min }$ it follows that the other two angles are both bounded by $\pi-\phi_{\min }$. Furthermore, from $\theta_{j}=2 \pi-\theta_{4}-\sum_{i=1, i \neq j}^{3} \theta_{i} \leq$ $2 \pi-\theta_{j}-2 \phi_{\min }$ it follows that $\theta_{j} \leq \pi-\phi_{\min }$ holds.

In the remainder we assume that quadrilaterals are subdivided in the way as explained in Theorem 3.2. Hence, the inner angles in the surface triangulation $\mathcal{F}_{h}$ are bounded by a constant $\theta^{*}<\pi$ that depends only on the stability (close to $\Gamma$ ) of the outer tetrahedral triangulation $\mathcal{T}_{h}$. In particular $\theta^{*}$ is independent of $h$ and of how $\Gamma_{h}$ intersects the outer triangulation $\mathcal{T}_{h}$.

4. Application in a finite element method. In this section, we use the maximum angle property of the surface triangulation to derive an optimal finite element interpolation result. On $\mathcal{F}_{h}$ we consider the space of linear finite element functions:

$$
V_{h}=\left\{v_{h} \in \mathcal{C}\left(\Gamma_{h}\right): v_{h} \in \mathcal{P}_{1}(T) \text { for all } T \in \mathcal{F}_{h}\right\} .
$$


This finite element space is the same as the one studied by Dziuk in [5], but an important difference is that in the approach in [5] the triangulations have to be shape regular. In general, the finite element space $V_{h}$ is different from the surface finite element space constructed in $[9,8]$.

Below we derive an approximation result for the finite element space $V_{h}$. Since the discrete surface $\Gamma_{h}$ varies with $h$, we have to explain in which sense $\Gamma_{h}$ is close to $\Gamma$. For this we use a standard setting applied in the analysis of discretization methods for partial differential equations on surfaces, e.g. [5, 4, 6, 7, 9].

Let $U:=\left\{x \in \mathbb{R}^{3} \mid \operatorname{dist}(x, \Gamma)<c\right\}$ be a sufficiently small neighborhood of $\Gamma$. We define $\mathcal{T}_{h}^{\Gamma}:=\left\{T \in \mathcal{T}_{h} \mid\right.$ meas $\left._{2}\left(T \cap \Gamma_{h}\right)>0\right\}$, i.e., the collection of tetrahedra which intersect the discrete surface $\Gamma_{h}$, and assume that $\mathcal{T}_{h}^{\Gamma} \subset U$. Let $d$ be the signed distance function to $\Gamma$, with $d<0$ in the interior of $\Gamma$,

$$
d: U \rightarrow \mathbb{R}, \quad|d(x)|:=\operatorname{dist}(x, \Gamma) \quad \text { for all } x \in U .
$$

Thus $\Gamma$ is the zero level set of $d$. Note that $\mathbf{n}_{\Gamma}=\nabla d$ on $\Gamma$. We define $\mathbf{n}(x):=\nabla d(x)$ for $x \in U$. Thus $\mathbf{n}$ is the outward pointing normal on $\Gamma$ and $\|\mathbf{n}(x)\|=1$ for all $x \in U$. Here and in the remainder $\|\cdot\|$ denotes the Euclidean norm on $\mathbb{R}^{3}$. We introduce a local orthogonal coordinate system by using the projection $\mathbf{p}: U \rightarrow \Gamma$ :

$$
\mathbf{p}(x)=x-d(x) \mathbf{n}(x) \quad \text { for all } x \in U .
$$

We assume that the decomposition $x=\mathbf{p}(x)+d(x) \mathbf{n}(x)$ is unique for all $x \in U$. Note that $\mathbf{n}(x)=\mathbf{n}(\mathbf{p}(x))$ for all $x \in U$. For a function $v$ on $\Gamma$, its extension is defined as

$$
v^{e}(x):=v(\mathbf{p}(x)), \quad \text { for all } x \in U .
$$

The outward pointing (piecewise constant) unit normal on $\Gamma_{h}$ is denoted by $\mathbf{n}_{h}$. Using this local coordinate system we introduce the following assumptions on $\Gamma_{h}$ :

$$
\begin{aligned}
& \mathbf{p}: \Gamma_{h} \rightarrow \Gamma \quad \text { is bijective, } \\
& \max _{x \in \Gamma_{h}}|d(x)| \lesssim h^{2}, \\
& \max _{x \in \Gamma_{h}}\left\|\mathbf{n}(x)-\mathbf{n}_{h}(x)\right\| \lesssim h .
\end{aligned}
$$

In (4.4)-(4.5) we use the common notation, that the inequality holds with a constant independent of $h$. In (4.5), only $x \in \Gamma_{h}$ are considered for which $\mathbf{n}_{h}(x)$ is well-defined. Using these assumptions, the following result is derived in [5].

Lemma 4.1. For any function $u \in H^{2}(\Gamma)$, we have, for arbitrary $T \in \mathcal{F}_{h}$ and $\tilde{T}:=\mathbf{p}(T):$

$$
\begin{aligned}
\left\|u^{e}\right\|_{0, T} & \sim\|u\|_{0, \tilde{T}}, \\
\left|u^{e}\right|_{1, T} & \sim|u|_{1, \tilde{T}}, \\
\left|u^{e}\right|_{2, T} & \lesssim|u|_{2, \tilde{T}}+h|u|_{1, \tilde{T}},
\end{aligned}
$$

where $A \sim B$ means $B \lesssim A \lesssim B$ and the constants in the inequalities are independent of $T$ and of $h$.

4.1. Finite element interpolation error. Based on the results in Lemma 4.1, the maximum angle property and the approximation results derived in [1] we easily 
obtain an optimal bound for the interpolation error in the space $V_{h}$. Consider the standard finite element nodal interpolation $I_{h}: C\left(\Gamma_{h}\right) \rightarrow V_{h}$ :

$$
\left(I_{h} v\right)(x)=v(x), \quad \text { for all } x \in \mathcal{V},
$$

with $\mathcal{V}$ the set of vertices of the triangles in $\Gamma_{h}$.

TheOREM 4.2. For any $u \in H^{2}(\Gamma)$ we have

$$
\begin{aligned}
\left\|u^{e}-I_{h} u^{e}\right\|_{L^{2}\left(\Gamma_{h}\right)} & \lesssim h^{2}\|u\|_{H^{2}(\Gamma)}, \\
\left\|u^{e}-I_{h} u^{e}\right\|_{H^{1}\left(\Gamma_{h}\right)} & \lesssim h\|u\|_{H^{2}(\Gamma)} .
\end{aligned}
$$

Proof. From standard interpolation theory we have

$$
\left\|u^{e}-I_{h} u^{e}\right\|_{L^{2}(T)} \lesssim h^{2}\left|u^{e}\right|_{2, T},
$$

where the constant in the upper bound is independent of (the shape of) $T$. Using the result in (4.8) and summing over $T \in \mathcal{F}$ proves the result (4.10). For the interpolation error bound in the $H^{1}$-norm we use the results from [1]. For the interpolation error bounds derived in that paper the maximum angle property is essential. From [1] we get

$$
\left\|u^{e}-I_{h} u^{e}\right\|_{H^{1}(T)} \lesssim h\|u\|_{H^{2}(T)} .
$$

Due to the maximum angle property the constant in the upper bound is independent of $T$. Using the results in Lemma 4.1 and summing over $T \in \mathcal{F}_{h}$ we obtain the result (4.11).

If one considers an $H^{1}(\Gamma)$ elliptic partial differential equation on $\Gamma$, the error for its finite element discretization in the surface space $V_{h}$ can be analyzed along the same lines as in [5]. Using the interpolation error bounds in Theorem 4.2 and bounding the geometric errors (due to approximation of $\Gamma$ by $\Gamma_{h}$ ) with the use of the assumptions (4.3)-(4.5) then results in optimal order discretization error bounds.

4.2. Conditioning of mass and stiffness matrix. Clearly the (strong) shape irregularity of the surface triangulation will influence the conditioning of the mass and stiffness matrices. Let $N$ be the number of vertices in the surface triangulation and $\left\{\phi_{i}\right\}_{i=1}^{N}$ the nodal basis of the finite element space $V_{h}$. The mass and stiffness matrices are given by

$$
\begin{aligned}
& \mathbf{M}=\left(m_{i j}\right)_{i, j=1}^{N}, \quad \text { with } \quad m_{i j}=\int_{\Gamma_{h}} \phi_{i} \phi_{j} d s, \\
& \mathbf{A}=\left(a_{i j}\right)_{i, j=1}^{N}, \quad \text { with } \quad a_{i j}=\int_{\Gamma_{h}} \nabla_{\Gamma_{h}} \phi_{i} \nabla_{\Gamma_{h}} \phi_{j} d s .
\end{aligned}
$$

We also need their scaled versions. Let $\mathbf{D}_{M}$ and $\mathbf{D}_{A}$ be the diagonals of $\mathbf{M}$ and $\mathbf{A}$, respectively. The scaled matrices are denoted by

$$
\mathbf{M}^{s}=\mathbf{D}_{M}^{-\frac{1}{2}} \mathbf{M} \mathbf{D}_{M}^{-\frac{1}{2}}, \quad \mathbf{A}^{s}=\mathbf{D}_{A}^{-\frac{1}{2}} \mathbf{A} \mathbf{D}_{A}^{-\frac{1}{2}} .
$$

From a simple scaling argument it follows that the spectral condition number of $\mathbf{M}^{s}$ is bounded uniformly in $h$ and in the shape (ir)regularity of the surface triangulation. For completeness we include a proof. 
THEOREM 4.3. The following holds:

$$
\frac{2}{\sqrt{2}+2} \leq \frac{\langle\mathbf{M v}, \mathbf{v}\rangle}{\left\langle\mathbf{D}_{M} \mathbf{v}, \mathbf{v}\right\rangle} \leq 4 \text { for all } \mathbf{v} \in \mathbb{R}^{N}, \mathbf{v} \neq 0 .
$$

Proof. The set of all vertices in $\mathcal{F}_{h}$ is denoted by $\mathcal{V}=\left\{\xi_{i} \mid 1 \leq i \leq N\right\}$. Let $\mathbf{v} \in \mathbb{R}^{N}$ and $v_{h} \in V_{h}$ be related by $v_{h}=\sum_{i=1}^{N} v_{i} \phi_{i}$, i.e., $v_{i}=v_{h}\left(\xi_{i}\right)$. Consider a triangle $T \in \mathcal{F}_{h}$ and let its three vertices be denoted by $\xi_{1}, \xi_{2}, \xi_{3}$. Using quadrature we obtain

$$
\begin{aligned}
\int_{T} v_{h}(s)^{2} d s & =\frac{|T|}{3}\left(\frac{1}{4}\left(v_{1}+v_{2}\right)^{2}+\frac{1}{4}\left(v_{2}+v_{3}\right)^{2}+\frac{1}{4}\left(v_{3}+v_{1}\right)^{2}\right) \\
& =\frac{|T|}{6}\left(v_{1}^{2}+v_{2}^{2}+v_{3}^{2}+v_{1} v_{2}+v_{2} v_{3}+v_{3} v_{1}\right) .
\end{aligned}
$$

Hence, $\int_{T} v_{h}(s)^{2} d s \leq \frac{|T|}{3} \sum_{i=1}^{3} v_{i}^{2}$ holds. From a sign argument it follows that at least one of the three terms $v_{1} v_{2}, v_{2} v_{3}$ or $v_{3} v_{1}$ must be positive. Without loss of generality we can assume $v_{1} v_{2} \geq 0$. Using $\left|v_{2} v_{3}+v_{3} v_{1}\right| \leq \frac{1}{\sqrt{2}}\left(v_{1}^{2}+v_{2}^{2}+v_{3}^{2}\right)$ we get

$$
\int_{T} v_{h}(s)^{2} d s \geq \frac{|T|}{6}\left(v_{1}^{2}+v_{2}^{2}+v_{3}^{2}-\frac{1}{\sqrt{2}}\left(v_{1}^{2}+v_{2}^{2}+v_{3}^{2}\right)\right)=\frac{|T|}{6(\sqrt{2}+2)}\left(v_{1}^{2}+v_{2}^{2}+v_{3}^{2}\right) .
$$

Note that $\langle\mathbf{M v}, \mathbf{v}\rangle=\int_{\Gamma_{h}} v_{h}(s)^{2} d s=\sum_{T \in \mathcal{F}_{h}} \int_{T} v_{h}(s)^{2} d s$, and thus we obtain, with $\mathcal{V}(T)$ the set of the three vertices of $T$,

$$
\frac{2}{\sqrt{2}+2} \frac{1}{12} \sum_{T \in \mathcal{F}_{h}}|T| \sum_{\xi \in \mathcal{V}(T)} v_{h}(\xi)^{2} \leq\langle\mathbf{M v}, \mathbf{v}\rangle \leq 4 \frac{1}{12} \sum_{T \in \mathcal{F}_{h}}|T| \sum_{\xi \in \mathcal{V}(T)} v_{h}(\xi)^{2} .
$$

We observe that

$$
\frac{1}{12} \sum_{T \in \mathcal{F}_{h}}|T| \sum_{\xi \in \mathcal{V}(T)} v_{h}(\xi)^{2}=\frac{1}{12} \sum_{i=1}^{N}\left|\operatorname{supp}\left(\phi_{i}\right)\right| v_{i}^{2}
$$

holds. From the definition of $\mathbf{D}_{M}$ it follows that

$$
\begin{aligned}
\left\langle\mathbf{D}_{M} \mathbf{v}, \mathbf{v}\right\rangle & =\sum_{i=1}^{N} \int_{\Gamma_{h}} \phi_{i}^{2} d s v_{i}^{2}=\sum_{i=1}^{N} v_{i}^{2} \sum_{T \in \operatorname{supp}\left(\phi_{i}\right)} \int_{T} \phi_{i}^{2} d s \\
& =\sum_{i=1}^{N} v_{i}^{2} \sum_{T \in \operatorname{supp}\left(\phi_{i}\right)} \frac{|T|}{12}=\frac{1}{12} \sum_{i=1}^{N}\left|\operatorname{supp}\left(\phi_{i}\right)\right| v_{i}^{2}
\end{aligned}
$$

Combination of the results in (4.14), (4.15) and (4.16) completes the proof.

The diagonally scaled stiffness matrix $\mathbf{A}^{s}$ has a one dimensional kernel due to the constant nodal mode. Thus, we consider the effective condition number $\operatorname{cond}\left(\mathbf{A}^{s}\right)=$ $\lambda_{\max }\left(\mathbf{A}^{s}\right) / \lambda_{2}\left(\mathbf{A}^{s}\right)$, where $\lambda_{2}$ is the minimal nonzero eigenvalue. We shall argue below that the condition number of $\mathbf{A}^{s}$ can not be bounded in general by a constant dependent exclusively on $\mathcal{T}_{h}$, but not on $\Gamma_{h}$. Indeed, assume a smooth closed surface $\Gamma$, with $|\Gamma|=1$, and a smooth function $u$ defined on $\Gamma$, such that $\left\|\nabla_{\Gamma} u\right\|_{L^{2}(\Gamma)}=\|u\|_{H^{2}(\Gamma)}=1$. Let $\Gamma_{h}$ be the zero level of the piecewise linear Lagrange interpolant of the signed 
distance function to $\Gamma$. Denote $u_{h}=I_{h} u^{e}$, as in Theorem 4.2, and $\mathbf{v}=\left(v_{1}, \ldots, v_{N}\right)^{T}$ is the corresponding vector of nodal values. From the result in (4.11) we obtain

$$
\langle\mathbf{A v}, \mathbf{v}\rangle=\left\|\nabla_{\Gamma_{h}} u_{h}\right\|_{L^{2}\left(\Gamma_{h}\right)}=1+O(h) .
$$

On the other hand, if there is a node $\xi$ in the volume triangulation $\mathcal{T}_{h}$ such that $\operatorname{dist}\left(\xi, \Gamma_{h}\right)<\varepsilon \ll 1$, then there can appear a triangle in $\mathcal{F}_{h}$ with a minimal angle of $O(\varepsilon)$. This implies that there is a diagonal element in $\mathbf{A}$ of order $O\left(\varepsilon^{-1}\right)$. Without lost of generality we may assume $A_{11}=O\left(\varepsilon^{-1}\right)$ and $v_{1}=1$. Thus we get

$$
\left\langle\mathbf{D}_{A} \mathbf{v}, \mathbf{v}\right\rangle \geq A_{11} v_{1}^{2}=O\left(\varepsilon^{-1}\right) .
$$

Comparing (4.17) and (4.18) we conclude that $\operatorname{cond}\left(\mathbf{A}^{s}\right) \geq O\left(\varepsilon^{-1}\right)$, with $\varepsilon \rightarrow 0$. Results of numerical experiments in the next section demonstrate that the blow up of $\operatorname{cond}\left(\mathbf{A}^{s}\right)$ can be seen in some cases.

One might also be interested in a more general dependence of the eigenvalues of $\mathbf{A}^{s}$ on the distribution of tetrahedral nodes in $\mathcal{T}_{h}$ in a neighborhood of $\Gamma_{h}$. To a certain extend this question is addressed in [8].

A strong growth of the condition number of the scaled stiffness matrix as in (4.17)(4.18) does not necessarily lead to a severe slown down of iterative solvers. This is illustrated and discussed in section 5 . One possibility to reduce the condition number deterioration sketched above is to 'glue' together nodes of $\mathcal{F}_{h}$ which have a distance less than $\varepsilon$ from each other, with some sufficiently small $\varepsilon$. We did not investigate this idea further.

5. Numerical experiment. In this section we present a few results of numerical experiments which illustrate the interpolation estimates from Theorem 4.2 and the conditioning of mass and stiffness matrices. Assume the surface $\Gamma$, which is the unit sphere $\Gamma=\left\{x \in \mathbb{R}^{3} \mid\|x\|=1\right\}$, is embedded in the bulk domain $\Omega=[-2,2]^{3}$. The signed distance function to $\Gamma$ is denoted by $d$. We construct a hierarchy of uniform tetrahedral triangulations $\left\{\mathcal{T}_{h}\right\}$ for $\Omega$, with $h \in\{1 / 2,1 / 4,1 / 8,1 / 16,1 / 32\}$. Let $d_{h}$ be the piecewise nodal Lagrangian interpolant of $d$. The triangulated surface is given by

$$
\Gamma_{h}=\bigcup_{T \in \mathcal{F}_{h}} T=\left\{x \in \Omega \mid d_{h}(x)=0\right\} .
$$

The corresponding finite element space $V_{h}$ consists of all piecewise affine functions with respect to $\mathcal{F}_{h}$, as defined in (4.1). For $h \in\{1 / 2,1 / 4,1 / 8,1 / 16,1 / 32\}$, the resulting dimensions of $V_{h}$ are $N=164,812,3500,14264,57632$, respectively. In agreement with the $2 \mathrm{D}$ nature of $\Gamma_{h}$, we have $N \sim h^{-2}$.

To illustrate the result of Theorem 4.2, we present the interpolation errors $\| u^{e}-$ $I_{h} u^{e} \|_{L^{2}\left(\Gamma_{h}\right)}$ and $\left|u^{e}-I_{h} u^{e}\right|_{1, \Gamma_{h}}$ for the smooth function

$$
u(x)=\frac{1}{\pi} x_{1} x_{2} \arctan \left(2 x_{3}\right)
$$

defined on the unit sphere, with $x=\left(x_{1}, x_{2}, x_{3}\right)^{T}$. The dependence of the interpolation errors on the number of degrees of freedom $N$ is shown in Figure 5.1 (left). We observe the optimal error reduction behavior, consistent with the estimates in (4.10), (4.11).

Further, for the same sequence of meshes we compute the spectral condition numbers of the mass matrix $\mathbf{M}$ and the diagonally scaled mass matrix $\mathbf{M}^{s}$. The 

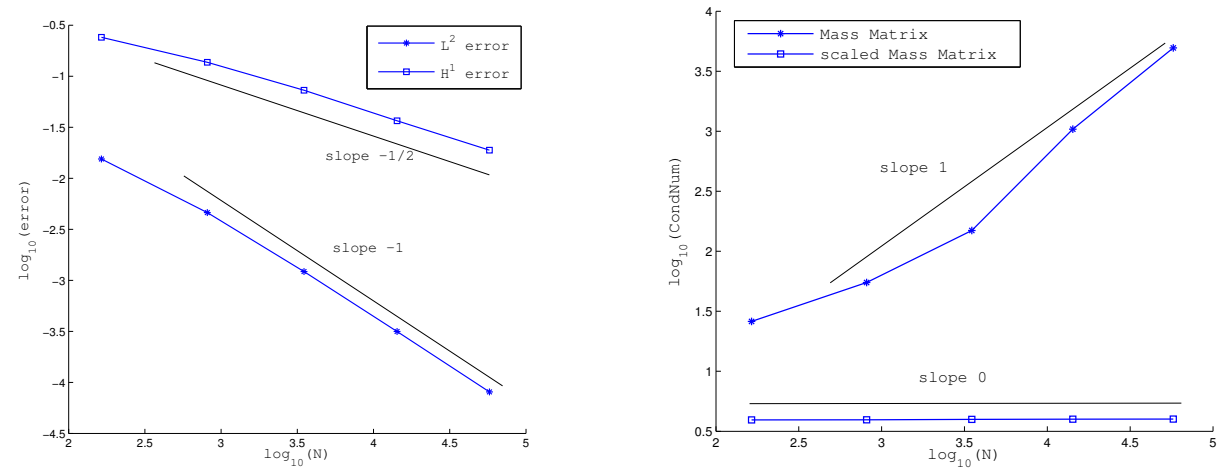

FiG. 5.1. Left: Interpolation error as a function of \# d.o.f.; Right: The condition number of the mass matrix as a function of of \# d.o.f.

dependence of the condition numbers on the number of degrees of freedom $N$ is illustrated in Figure 5.1 (right). As was proved in Theorem 4.3, the scaled mass matrix has a uniformly bounded condition number.

We discussed in section 4.2 that the situation with the effective conditioning of the scaled stiffness matrix is more delicate. To illustrate numerically the dependence of $\operatorname{cond}\left(\mathbf{A}^{s}\right)$ on the position of $\Gamma_{h}$ with respect to the outer triangulation, we perform the following series of experiments. Let $\Gamma$ be the boundary of the unit sphere with the center located in $\left(0,0, z_{c}\right)$. The discrete surface $\Gamma_{h}$ is defined as described above, induced by the uniform outer triangulations. Now we compute the effective condition number of $\mathbf{A}^{s}$ varying both the mesh size of the outer grid $h$ and the sphere's center location $z_{c}$. Results presented in Table 5.1 show the strong dependence of $\operatorname{cond}\left(\mathbf{A}^{s}\right)$ on the sphere location. The dramatic decrease of cond $\left(\mathbf{A}^{s}\right)$ for $z_{c}=0$ happens because in that case certain nodes of $\mathcal{T}_{h}$ lie exactly on $\Gamma_{h}$. Otherwise these nodes result in triangles in $\mathcal{F}_{h}$ that may have very sharp angles which then lead to the blow up of $\operatorname{cond}\left(\mathbf{A}^{s}\right)$. We also note that the interpolation errors (not shown) were (almost) independent on the position of $\Gamma_{h}$ with respect to the outer triangulation.

In Table 5.1 we also show the total number of iterations for the diagonally preconditioned CG method applied to solve $\mathbf{A x}=\mathbf{b}$ up to the relative residual tolerance of $10^{-10}$. Note that the number of iterations depends on $z_{c}$ in a much less dramatic way than $\operatorname{cond}\left(\mathbf{A}^{s}\right)$ does. The explanation of this observation is the following: Few nodes 'close' to $\Gamma_{h}$ lead to a small number of outliers in the spectrum of $\mathbf{A}^{s}$. The small number of outliers in the spectrum is well known to result only in temporal stagnation period(s) in the convergence history of a Krylov subspace method rather than in a permanent low convergence rate, see, e.g., [10] and references cited therein. This phenomenon is illustrated in Figure 5.2, where we show the convergence histories of the $\mathrm{CG}$ method for $\mathbf{A x}=\mathbf{b}$ with the diagonal preconditioner and different values of $z_{c}$.

Acknowledgments. This work has been supported in part by the DFG through grant RE1461/4-1 and the Russian Foundation for Basic Research through grants 1201-91330, 12-01-00283. 


\begin{tabular}{l|cc|cc|cc} 
& \multicolumn{2}{|c|}{$h=1 / 4$} & \multicolumn{2}{c|}{$h=1 / 8$} & \multicolumn{2}{c}{$h=1 / 16$} \\
\hline$z_{c}$ & cond $\left(\mathbf{A}^{s}\right)$ & $N_{C G}$ & cond $\left(\mathbf{A}^{s}\right)$ & $N_{C G}$ & cond $\left(\mathbf{A}^{s}\right)$ & $N_{C G}$ \\
\hline 0.008 & $2.96 \mathrm{e}+04$ & 178 & $2.15 \mathrm{e}+04$ & 478 & $4.78 \mathrm{e}+04$ & 1362 \\
0.002 & $4.69 \mathrm{e}+05$ & 187 & $3.30 \mathrm{e}+05$ & 403 & $2.13 \mathrm{e}+05$ & 1083 \\
0.0005 & $7.49 \mathrm{e}+06$ & 200 & $5.27 \mathrm{e}+06$ & 429 & $3.35 \mathrm{e}+06$ & 880 \\
0.00025 & $3.00 \mathrm{e}+07$ & 205 & $2.10 \mathrm{e}+07$ & 440 & $1.34 \mathrm{e}+07$ & 906 \\
0.00005 & $7.49 \mathrm{e}+08$ & 236 & $5.27 \mathrm{e}+08$ & 497 & $3.34 \mathrm{e}+08$ & 949 \\
0.00001 & $3.66 \mathrm{e}+07$ & 202 & $8.68 \mathrm{e}+07$ & 514 & $3.95 \mathrm{e}+08$ & 1020 \\
0 & $4.62 \mathrm{e}+02$ & 56 & $2.45 \mathrm{e}+03$ & 142 & $1.10 \mathrm{e}+04$ & 295 \\
\hline
\end{tabular}

Dependence of the effective condition number of $\mathbf{A}^{s}$ and the number of $C G$ steps $\left(N_{C G}\right)$ on the position of $\Gamma_{h}$

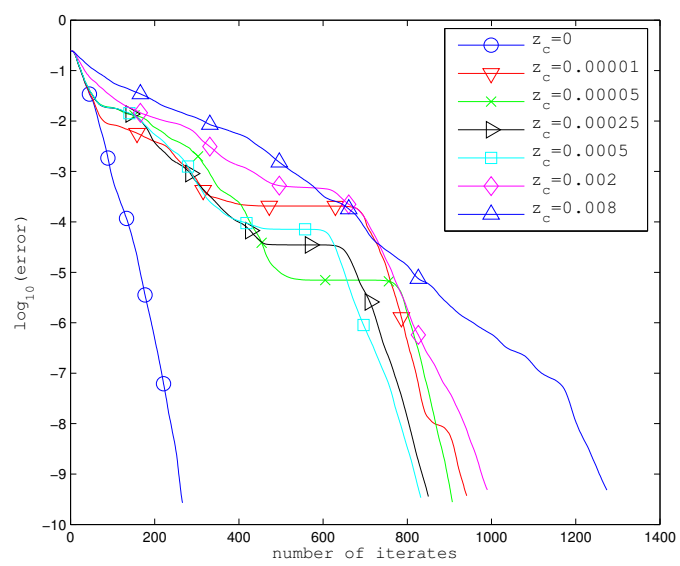

FIG. 5.2. Convergence history of the diagonally preconditioned $C G$ method for $\mathbf{A x}=\mathbf{b}$, with $h=1 / 16$ and different values of $z_{c}$.

[1] I. Babuška and A. K. Aziz. On the angle condition in the finite element method. SIAM J. Numer. Anal., 13:214-226, 1976.

[2] P. Ciarlet. The Finite Element Method for Elliptic Problems. North-Holland, Amsterdam, 1978.

[3] A. Demlow. Higher-order finite element methods and pointwise error estimates for elliptic problems on surfaces. SIAM J. Numer. Anal., 47:805-827, 2009.

[4] A. Demlow and G. Dziuk. An adaptive finite element method for the Laplace-Beltrami operator on implicitly defined surfaces. SIAM J. Numer. Anal., 45:421-442, 2007.

[5] G. Dziuk. Finite elements for the Beltrami operator on arbitrary surfaces. In S. Hildebrandt and R. Leis, editors, Partial differential equations and calculus of variations, volume 1357 of Lecture Notes in Mathematics, pages 142-155. Springer, 1988.

[6] G. Dziuk and C. Elliott. Finite elements on evolving surfaces. IMA J. Numer. Anal., 27:262$292,2007$.

[7] G. Dziuk and C. Elliott. $L^{2}$-estimates for the evolving surface finite element method. SIAM J. Numer. Anal., 2011. to appear.

[8] M. A. Olshanskii and A. Reusken. A finite element method for surface PDEs: matrix properties. Numer. Math., 114:491-520, 2009.

[9] M. A. Olshanskii, A. Reusken, and J. Grande. An Eulerian finite element method for elliptic equations on moving surfaces. SIAM J. Numer. Anal., 47:3339-3358, 2009.

[10] M. A. Olshanskii and V. Simoncini. Acquired clustering properties and solution of certain saddle point systems. SIAM J. Matrix Anal. Appl., 31:2754-2768, 2010.

[11] J.-J. Xu and H.-K. Zhao. An Eulerian formulation for solving partial differerential equations along a moving interface. J. Sci. Comput., 19:573-594, 2003. 